\title{
El estudio científico de los megalitos (1). La geoarqueología
}

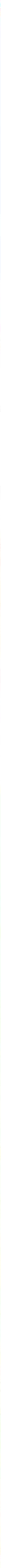




\section{INTRODUCCIÓN}

La geoarqueología es una disciplina mixta que combina objetivos y métodos de la arqueología y de las ciencias de la tierra (geografía física geología, sedimentología, geofísica y edafología, etc.). De forma general su propósito podría describirse como la reconstrucción de los medios físicos en los que se desenvolvieron las comunidades humanas estudiadas arqueológicamente, así como la reconstrucción de las relaciones de interacción establecidas entre las comunidades de seres humanos y el medio físico que ocuparon, con especial atención a su evolución en el tiempo. Aplicada al estudio del megalitismo, la geoarqueología se centra en problemas como la aproximación al contexto geográfico y geológico de las construcciones megalíticas en el marco de la escala local y regional, la caracterización geológica y petrológica de los materiales constructivos, la identificación de los lugares de aprovisionamiento (canteras) de dichos materiales constructivos y el análisis estructural y geotécnico de las propias construcciones en sí, que pueden ayudar a determinar numerosos aspectos de su génesis y uso (orden de colocación de los bloques de piedra, y por tanto posibles fases constructivas, inversión de esfuerzo y trabajo en el transporte y colocación de dichos bloques, según su tamaño o accesibilidad, técnicas arquitectónicas empleadas, etc.). Los datos geoarqueológicos son asimismo de vital importancia para cualquier estrategia cualificada de tratamiento, consolidación, restauración y mantenimiento de monumentos megalíticos.

Por tanto, desde una perspectiva científica novedosa derivada de la aplicación de los métodos de las ciencias de la tierra y otras disciplinas al uso, en esta colaboración se propone un ejemplo de cómo la geoarqueología puede contribuir al avance del estudio y gestión patrimonial de las construcciones megalíticas, así como al enriquecimiento del debate relativo a las diversas estrategias de investigación (y por extensión, de gestión) de los sitios de carácter arqueológico. Hemos partido de la experiencia desarrollada en el Conjunto Arqueológico Dólmenes de Antequera (Málaga) en el periodo 2005-2008 por un equipo de carácter interdisciplinar de la Universidad de Granada, conformado por arqueólogos, geólogos, geofísicos y matemáticos. El carácter fuertemente interdisciplinar de esta investigación, ha dado lugar a un estudio geoarqueológico de los dólmenes de Menga y Viera, y el tholos de El Romeral, realizado desde perspectivas diferentes a la investigación arqueológica tradicional, donde nuevos métodos y técnicas de análisis han podido responder a preguntas de difícil respuesta para la arqueología tradicional.

\section{ESTUDIO GEOLÓGICO: CARTOGRAFÍA GEOLÓGICA LOCAL, CARACTERIZACIÓN DE LOS BLOQUES CONSTRUCTIVOS E IDENTIFICACIÓN DE SU PROCEDENCIA}

La clasificación, estudio, reconocimiento in situ y cartografiado del medio geológico, desde diversas perspectivas (sedimentológica, tectónica, estratigráfica, geomorfológica, hidrogeológica, etc.) permite construir una representación espacial bastante exacta de las diversas litologías del medio en el que una construcción megalítica fue erigida. Clasificar las diversas litologías presentes en cada una de las grandes construcciones de la necrópolis megalítica antequerana desde estas perspectivas ha constituido el primero de los pilares fundamentales de esta investigación, proporcionando un caudal de información cualificada, abundante y diversificada. Un objetivo principal de este análisis ha sido contrastar la existencia de posibles relaciones entre los materiales líticos empleados en el sistema constructivo de las sepulturas y los afloramientos rocosos naturales espacialmente representados, tanto en el entorno como en las proximidades de la necrópolis. El estudio de estas relaciones entre necrópolis y afloramientos nos conduce al examen de problemas tales como las distancias desde la fuente de suminis- 
tro hasta el emplazamiento del dolmen, la tecnología de extracción de bloques en las canteras, o la tecnología empleada para el transporte de los ortostatos y losas de cobertura hasta su mismo emplazamiento final.

Para ello se ha realizado un estudio geológico local de los entornos de la ciudad de Antequera con la creación ex novo de una cartografía geológica de detalle a escala 1:10.000. Se ha obtenido una litoteca de referencia cuyo sistema de muestreo ha sido georeferenciado mediante GPS. Se ha elaborado una memoria explicativa de la cartografía geológica. Además del estudio petrográfico y petrológico, se han elaborado análisis micropaleontológicos

Una vez obtenida la cartografía geológica de detalle del entorno de la necrópolis, se ha elaborado un estudio detallado de la geología de las tres sepulturas, incidiéndose especialmente en el dolmen de Menga por su singularidad y relevancia. Para ello se han analizado los siguientes elementos: análisis de los componentes estructurales mediante estudio litológico, características paleontológicas y micropalentológicas (calcarenitas, calciruditas bioclásticas, etc.), estudio mineralógico, alteraciones químicas (precipitados de óxidos, carbonatos, etc.), fracturas en losas de cubierta y ortostatos.

El estudio litológico realizado en el dolmen de Menga, ha permitido diferenciar a grandes rasgos tres tipos distintos de rocas empleadas en sus ortostatos: calcarenitas y calcirruditas bioclásticas poco cementadas (Grainstone), así como brecha plana poligénica con matriz calcárea muy cementada (Packstone) y por último un microconglomerado con bivalvos. Al respecto hay que decir que, los ortostatos, losas y pilares realizados con estas areniscas bioclásticas, pueden presentar características mixtas entre calcarenitas y calcirruditas.

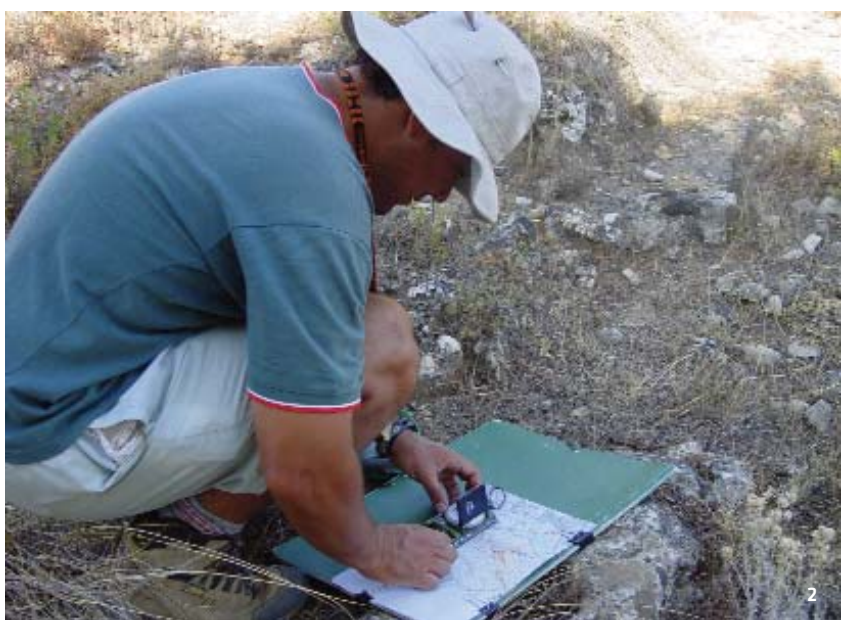

Sin embargo, por los requerimientos de nuestro trabajo (origen de estas materias líticas, características de estas rocas, etc.), fue necesario el estudio de la situación estratigráfica de las mismas en su contexto geológico, génesis, medio sedimentario, facies sedimentarias, paleogeología, paleoclimatología, etc. Para todo esto, se estudiaron la paleontología y la micropaleontología de cada una de los componentes estructurales del sepulcro tanto a visu como a lámina delgada. Este último estudio, se realizó en un número de muestras lo más representativo posible teniendo en cuenta la heterogeneidad lítica que existe en el dolmen de Menga.

Los resultados fueron que los componentes estructurales del sepulcro megalítico correspondientes a calcarenitas y calcirruditas bioclasticas, pertenecían a dos facies paleogeográficas bien diferenciadas. Por un lado las correspondientes a las Facies de Factoría (prácticamente la totalidad de las estructuras composicionales que constituyen el dolmen con características de areniscas), y por otro lado la que corresponde a las Facies de Foreshore.

Una vez realizada la caracterización geológica precisa de cada uno de los bloques de piedras constitutivos de la arquitectura del dolmen de Menga, y conociéndose la cartografía geológica de la zona, ha sido posible abordar el problema de la procedencia de los materiales constructivos. Los resultados obtenidos en el estudio geológico detallado nos han conducido finalmente hacia el área de captación y explotación de estas rocas de Edad Tortoniense, descartándose la hipótesis previamente manejada por la que se pensaba que provenían de una cantera relativamente cercana, situada en el Cerro de la Cruz.

En el caso de los dólmenes de Menga y Viera, los materiales proceden de una paleogeografía más Ilana que en la actualidad, próxima al emplazamiento de los dólmenes, constituida por calcarenítas y materiales brechoides, desechando la hipótesis de la procedencia de éstos del Cerro de la Cruz, sobre una topografía mas elevada y de características litológicas que si son parecidas a la vista, no son exactamente las mismas que las empleadas en las construcciones de Menga y Viera.

\section{ESTUDIO ESTRUCTURAL Y GEOTÉCNICO}

Además del estudio geológico integral del dolmen de Menga y del entorno de la necrópolis megalítica antequerana, se ha llevado a cabo un estudio estructural y geotécnico completo de la primera de estas construcciones. Para ello se han realizado mediciones y cálculos matemáticos sobre todos y cada uno de los elementos líticos que componen el dolmen, determinándose volúmenes y haciéndose los cálculos relativos al sistema constructivo. 

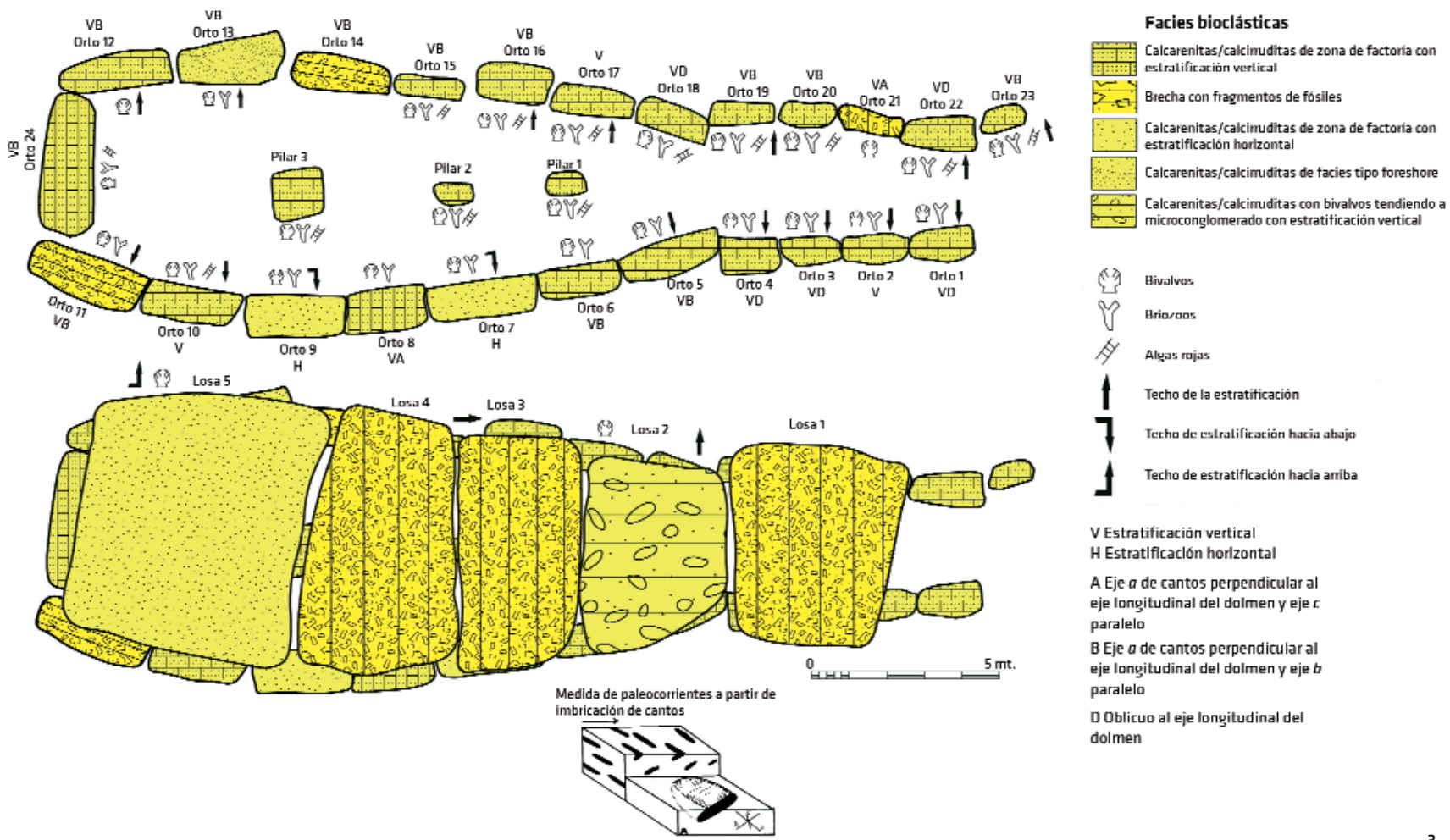

Las dimensiones del monumento mantienen unas proporciones casi perfectamente regulares a partir de las partes visibles del mismo: altura visible y anchura visible. Los ortostatos y las losas que conforman la cubierta están bastante alisadas y se mantienen en un plano paralelo al suelo, sin inclinaciones laterales. Y puesto que el suelo mantiene una pequeña inclinación, existe una variación de las alturas visibles en tres partes, lo que permite mantener el paralelismo de las losas de la cubierta. Existen tres partes respecto a las alturas visibles que constituyen conjuntos independientes formada por: los 5 primeros ortostatos a partir del central; los ortostatos comprendidos entre el seis y el diez; y una parte muy residual distinta del resto del dolmen formada por los dos pequeños ortostatos del final.

Los análisis previos indican que otras variables no visibles no están previamente determinadas. Así, el grosor, la anchura, la altura total (en los casos en los que se ha podido establecer) son muy variables. Además, la parte oculta de los ortostatos verticales no tienen relación con respecto a la parte visible, es decir, son independientes. Este hecho permite establecer la hipótesis de que en la construcción del monumento, el ortostato se acerca a la zona de colocación e, in situ, se mide la parte visible para que esté en concordancia con el resto ya colocado, y se determina la parte que estará oculta. Posteriormente se excava el cimiento en que se situará el ortostato para que encaje de forma precisa la parte oculta del mismo; de esta forma se consigue que la parte visible sea la correcta para mantener el diseño general del monumento.

Además, tan importante como el contenido, es decir los elementos arquitectónicos, lo es el continente, la estructura tumular, responsable de la estabilidad del dolmen, pero muy alterada por prácticas agropecuarias tradicionales, expolios, antiguas excavaciones arqueológicas, alteración de la forma original por construcciones de caminos de vehículos y peatonales, etc. Fundamentalmente dos han sido los métodos empleados en el estudio de la estructura tumular de Menga: prospecciones geofísicas y sondeos estratigráficos con metodología arqueológica.

Las distintas técnicas de prospección geofísica (resistividad, magnetométrica, georadar) permiten realizar indagaciones no destructivas en el subsuelo. Los perfiles de reflexión, la modalidad más usual de adquisición de datos georadar, consisten en desplazar una antena que emite pulsos de energía electromagnética en la banda de radiofrecuencia. Dichos pulsos viajan hacia el interior del terreno en forma de ondas electromagnéticas y parte de la energía que transportan se refleja en las 
4. Peso de los bloques de piedra de Menga / Fuente: ReAlizado a PARTIR DE CARrión MÉnDEZ; LOZANo RODRÍCueZ; GARCÍA GONZÁlez, et al., 2006

5. Macrofotografía de una calcarenita bioclástica que muestra restos de pectínidos de edad Tortoniense / Fuente: C. A. Dólmenes de ANTEQUera

7. Trabajos de topografía y georreferenciación de la estructura tumular del dolmen de Menga / FUENTE: C. A. DóLMENES DE ANTEQUERA
6. Macrofotografía de calcarenita con retos de briozoos, fauna propia de mares cálidos y poco profundos de edad Tortoniense / FUENTE: C. A. DóLMENES DE ANTEQUERA

8. Interior del dolmen de Menga. Materiales litoestratigráficos donde se cimenta esta megaconstrucción / FUENTE: C. A. DóLMENES DE ANTEQUERA
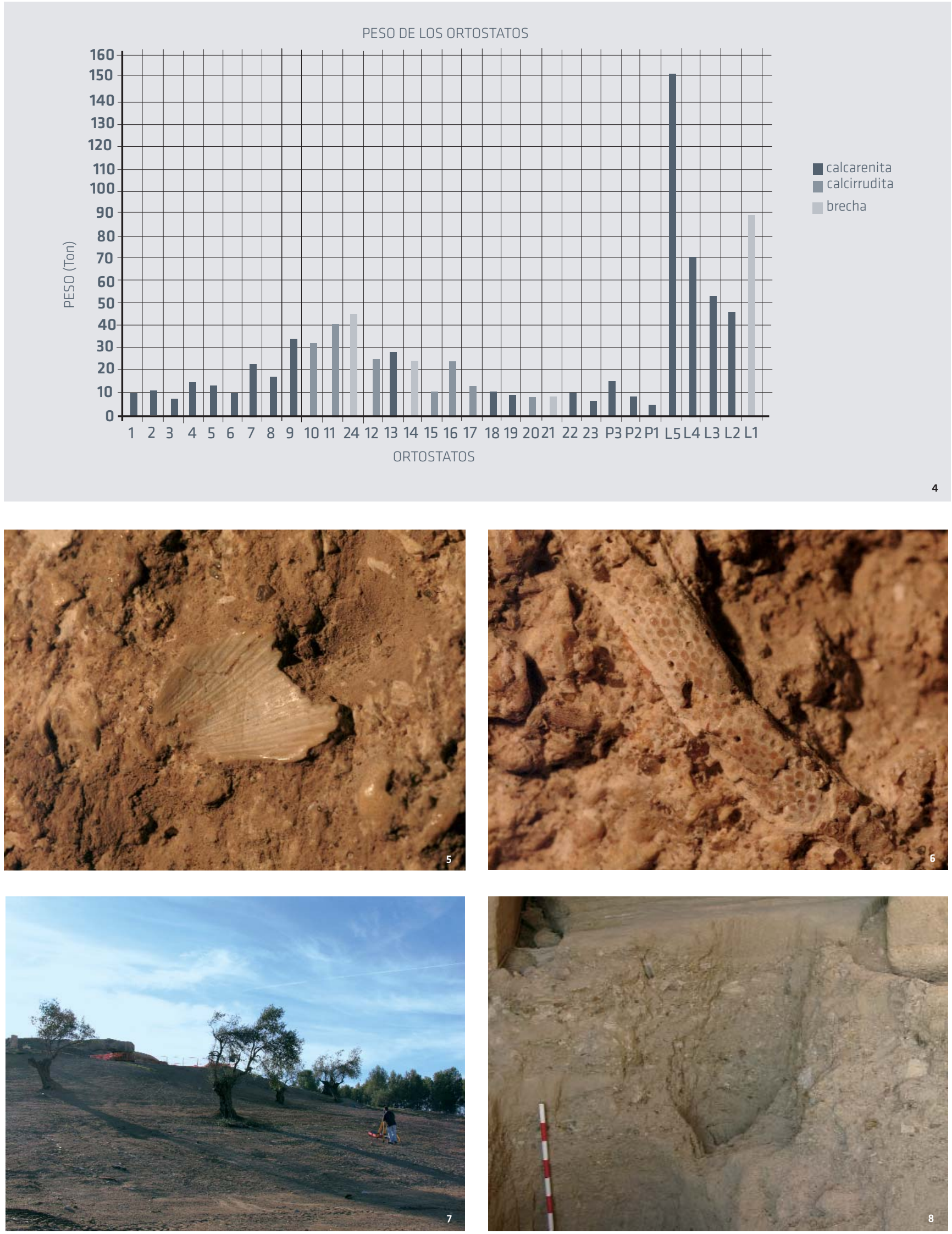
superficies de discontinuidad electromagnética encontrada, originada por cambios de materiales y también por cambios en el contenido en agua dentro de unos mismos materiales. Para que estas ondas se reflejen es necesario que exista un contraste notable en un parámetro denominado constante dieléctrica entre los dos materiales en contacto. Si esto no ocurre, no hay reflexión; de modo que puede haber un contacto entre dos materiales de aspecto radicalmente diferente a simple vista, pero que por tener valores similares de la constante dieléctrica (parámetro antes aludido) no devuelve energía y son tomados por un material único. Lo contrario también puede ocurrir: un mismo material puede tener un distinto valor de constante dieléctrica en función de su contenido en fluidos (agua o contaminantes líquidos). La profundidad de penetración y la resolución (detección de los cuerpos) dependen de las características del terreno y de la antena utilizada. La profundidad disminuye al aumentar la conductividad del terreno; también lo hace al aumentar la frecuencia central de la antena. Si se emplean antenas de mayor frecuencia se obtiene más resolución y menor profundidad de penetración, y a la inversa al disminuir la frecuencia. A pesar de que los criterios de interpretación de esta prospección geofísica se han establecido en base a toda la información disponible (perfiles de contraste, bibliografía, información arqueológica, etc.), los radargramas presentados en este estudio representan un modelo del terreno y no pueden llegar nunca a un nivel de precisión como el que aporta una excavación arqueológica.

Para contrastar los resultados de la prospección geofísica con radar se ha realizado un sondeo arqueológico estratigráfico, radial a la estructura circular del túmulo y que en su parte más profunda alcanzó los cuatro metros. En este sondeo se pudo determinar, entre otras consideraciones, el sistema constructivo del túmulo, materiales empleados de las mismas propiedades que las manufacturas de losas y ortostatos, además de arenas carbonatadas. A partir de este sondeo se ha podido establecer que el túmulo en sí mantiene una técnica constructiva basada en la colocación de niveles de bloques de piedra de tamaño medio entre los que se alternan rellenos terrígenos. De esta forma se le dota de mayor consistencia a la construcción, minimizando los efectos que la erosión pudiera haber provocado en un túmulo exclusivamente realizado con aporte de tierra, y facilita el drenaje del agua de lluvia hacia los laterales del túmulo. Las características de la litología empleada, fundamentalmente calcarenita, favorece aun más esta labor de drenaje. La gran cantidad de bloques que se han utilizado para conformar el túmulo supone una fuerte actividad de cantería y extracción de material que se une a la de los propios elementos estructurales del dolmen. El relleno de la masa tumular muestra al menos dos fases diferentes en la construcción del túmulo, que parecen coincidir con las fases de construcción de la estructura arquitectónica del dolmen. Con este conocimiento se pueden aportar datos desde la geoarqueología para los futuros planes de recuperación de la masa tumular original, empleando las mismas técnicas constructivas y materiales que los constructores del pasado.

Por otra parte, el estudio geotécnico ha tenido como objetivos caracterizar las propiedades mecánicas del material sobre el que se asientan los ortostatos, determinar la carga de hundimiento y los asientos inducidos, determinar las características físicas de los ortostatos y losas de cubierta (al menos de sus litologías representativas), entre las que podemos destacar la densidad aparente, la densidad seca, el peso específico de las partículas sólidas, la porosidad, la humedad y el grado de saturación, estudiar las propiedades mecánicas de los ortostatos y losas de cubierta (tales como la resistencia a compresión simple, la resistencia a tracción, módulo de elasticidad, etc.), determinar las solicitaciones de esfuerzos a las que están sometidos los distintos soportes, debido tanto a su propio peso como a la sobrecarga del material que conforma el túmulo, y explicar el origen de fracturas que presentan algunas piedras en la actualidad (fundamentalmente las losas de cubierta).

El estudio geotécnico ha permitido establecer algunas conclusiones de interés. Así, por un lado, queda establecido que la elevada capacidad portante del terreno y las pequeñas presiones ejercidas por los ortostatos sobre el mismo han asegurado la estabilidad del dolmen a lo largo de los siglos. En cuanto al análisis estructural cabe decir que las grietas observadas en las losas 1 y 2 no se han producido por el momento flector debido al propio peso de la roca y a la sobrecarga de tierras sobre la misma, puesto que la tracción inducida por dicho momento es bastante inferior a la resistencia a tracción de la roca. En el resto de las losas de cubierta la tracción ocasionada por el momento es menor, pero próximo a la resistencia a tracción de la roca e incluso pudo haber sido superado en el pasado con una mayor sobrecarga de tierras en el túmulo. Tal era la conciencia de tal efecto que por esta razón se colocaron los pilares centrales de forma que dicho momento sufriera una reducción considerable. Las grietas que se observan en los losas 1 y 2 se han debido producir por movimientos sísmicos con un grado de intensidad superior a 8 . Al efecto de estos movimientos sísmicos hay que añadir el momento flector máximo que actúa permanentemente en el eje central de las losas, el cambio de momentos flectores que sufrieron estas losas cuando se eliminó el soporte central y la presencia de discontinuidades internas. Todas estas conclusiones son de especial relevancia en el tratamiento de consolidación, mantenimiento y restauración de esta gran construcción megalítica.

\section{Bibliografía}

CARRIÓN MÉNDEZ, F.; LOZANO RODRÍGUEZ, J. A.; GARCíA GONZÁLEZ et al. (2006) Estudio Geoarqueológico de los Sepulcros Megalíticos de Cueva de Menga, Viera y Romeral (Antequera, Málaga) [literatura gris]. Granada: Universidad de Granada, 2006 